\title{
Retraction: Cooling flexural modes of a mechanical oscillator by magnetically trapped Bose-Einstein-condensate atoms [Phys. Rev. A 96, 063813 (2017)]
}

\author{
Donghong $\mathrm{Xu}$ and Fei Xue
}

(Received 11 November 2018; published 30 November 2018)

DOI: 10.1103/PhysRevA.98.059902

Recently, the authors have found a flaw in the basic modeling of the system presented in this paper. In modeling a hybrid system of a mechanical oscillator and BEC atoms, the hyperfine states $|1,-1\rangle$ and $|1,0\rangle$ of ${ }^{87} \mathrm{Rb}$ are chosen as eigenstates in the Jaynes-Cummings Hamiltonian, which will not cool the flexural modes of the mechanical oscillator.

The proper choice of eigenstates of ${ }^{87} \mathrm{Rb}$ in the Jaynes-Cummings Hamiltonian should be the hyperfine states $|1,-1\rangle$ and $|2,-2\rangle$. When using the hyperfine states $|1,-1\rangle$ and $|2,-2\rangle$ of ${ }^{87} \mathrm{Rb}$ as eigenstates in the Jaynes-Cummings Hamiltonian, the theoretical analyses given in Secs. II and III are still valid.

However, this new choice of eigenstates will greatly affect the methods of cooling the mechanical oscillators. To match the energy of the hyperfine states $|1,-1\rangle$ and $|2,-2\rangle$, the frequency of the mechanical oscillators is in the order of $6 \mathrm{GHz}$, instead of a tunable frequency range from a few $\mathrm{MHz}$ to a few hundred $\mathrm{MHz}$ in this paper. Also, the coupling strength between the mechanical oscillator and ${ }^{87} \mathrm{Rb}$ atoms is also reduced. Therefore, Sec. IV should be reconsidered. The cooling efficiency for mechanical oscillators in this study is greatly reduced. Since the cooling efficiency is an indispensable part of this paper, we wish to retract this paper to avoid misleading the readership. We regret the error. 